\title{
Adverse effects of whole blood donation among voluntary blood donors in Jos, Nigeria
}

\author{
Damulak Obadiah Dapus ${ }^{1, ~ *}$, Egesie Ochaka Julie ${ }^{1}$, Chetle Ladi' ${ }^{2}$, Thomas Margaret ${ }^{2}$ \\ ${ }^{1}$ Dept of Haematology \& Blood Transfusion, Faculty of Medical Sciences, University of Jos, Jos, Nigeria \\ ${ }^{2}$ National Blood Transfusion Service, North Central Zonal Centre, Jos, Nigeria
}

Email address:

ddamulak@yahoo.com (Damulak O. D.)

\section{To cite this article:}

Damulak Obadiah Dapus, Egesie Ochaka Julie, Chetle Ladi, Thomas Margaret. Adverse Effects of Whole Blood Donation among Voluntary Blood Donors in Jos, Nigeria. Clinical Medicine Research. Vol. 4, No. 1, 2015, pp. 6-10. doi: 10.11648/j.cmr.20150401.12

\begin{abstract}
Introduction: Despite collection of blood from apparently healthy individuals for allogeneic transfusion, blood givers are often faced with untoward reactions during or after blood donation. Aim: This study was to determine the rate of adverse reactions among voluntary blood donors at the National Blood Transfusion Service in Jos. Methods: All blood donors recruited by the centre between October 2012 and September 2014 were counseled and consent to participate in the research was obtained prior to donation. The age, sex, weight and blood pressure and the venue of donation were documented. The type of donation reaction during and or after donation were timed and documented. The haemoglobin level and haemoglobin phenotypes were determined. Results: Eleven thousand six hundred and fifty-five (63\%) male and 37\% females were studied. The overall rate of adverse effects was $2.05 \%$ with significantly higher rate of occurrence among donors aged $18-25$ years $(\mathrm{p}<0.0001)$, female donors $(\mathrm{p}=0.0001)$, weight $40-49 \mathrm{Kg}(\mathrm{p}=0.001)$, blood group $\mathrm{B}(\mathrm{p}=0.002)$ haemoglobin phenotype AA $(\mathrm{P}=0.001)$. The rate of adverse reactions was also higher among first-time donors $(\mathrm{p}=0.002)$, indoor donations $(\mathrm{p}=0.001)$. All adverse effects documented in our donors occurred during donation (4\%) and within $21(96 \%)$ after donation. Dizziness affected $90 \%$ while severe reaction (faint) occurred in 3\% of adverse reactors. Ingested meal was the vomits of all donors who vomited. Conclusion: Though adverse effects to blood donation is low in our centre, there is need for preparedness, donor education, prediction and mitigation of occurrences.
\end{abstract}

Keywords: Adverse Effects, Voluntary, Blood Donation, Jos, Nigeria

\section{Introduction}

Blood donation and transfusion constitutes part of daily effective medical practice as there are no efficient substitutes to blood when needed. Blood is obtained from humans for allogeneic transfusion. Despite the fact that blood is sourced from apparently healthy individuals some adverse reactions usually occur during or after blood donation.

Reports abound from various researchers on the adverse reactions to blood donation. Newman in 2003 reported a 36\% occurrence of adverse blood donation reactions among his blood donors. He found fatigue in $7.8 \%$, vaso-vagal symptoms in $5.3 \%$, and bruises in $22.7 \%$. Other effects were nausea and vomiting in $1.1 \%$, soreness in $10 \%$ and haematoma in $1.7 \%$ blood donors. ${ }^{1}$ He noticed a higher rate of $48 \%$ adverse effects among female than $23 \%$ prevalence in male blood donors. He further reported higher rate of $47 \%$ among first time blood donors than a lower $36 \%$ among repeat donors. ${ }^{1}$ In another study, he reported the occurrence of adverse effects of blood donation in a third of American donors annually with bruises $23 \%$, sore arm $10 \%$, fatigue $80 \%$ and vaso vagal symptoms $7 \%{ }^{2}$ In yet another research he found bruises in $23 \%$, sore arms in $10 \%$, vaso vagal reactions in $7 \%$, fatigue in $8 \%$, nerve irritation in $0.9 \%$, syncope in $0.1-0.3 \%$, arterial puncture in $0.01 \%$ with $0.033 \%$ of such reaction compelling donors to seek medical care. ${ }^{3}$ Newman et al also reported the frequency of adverse effects of blood donation to include bruise in $22.7 \%$, sore arm in $10 \%$, fatigue in $7.8 \%$, and other donation reactions in $7.0 \%$ of 1000 interviewed American blood donors. ${ }^{4}$

Crocco and others while studying adverse effects of blood donation in Italy reported an overall lower prevalence of 1.2\% adverse donation effects among their volunteer blood donors. They further found that $1.08 \%$ of the observed effects were mild, while $0.02 \%$ were severe. Severe effects included fainting and convulsion. ${ }^{5}$ The high prevalence of adverse donation effects were not replicated in an Indian study where Pathak and his colleagues reported a low $0.6 \%$ prevalence of 
adverse reactions among their blood donors. Pre-syncopal symptoms of mild intensity accounted for $70 \%$ of the reactions, haematoma $0.07 \%$, sore arm $0.05 \%$, syncopal minor $0.05 \%$ and syncopal major $0.005 \%{ }^{6}$

Eder and others reported varying prevalence of blood donation reactions in their blood donors. They found a prevalence of $10.7 \%$ among donors aged 16-17 years, $8.3 \%$ in 18-19 years and $2.8 \%$ in donors twenty year old and above. ${ }^{7}$ A similar study led by Wiltbuk reported the occurrence of faints and prefaints reactions in whole blood donors. They found higher rate of occurrence among younger and first time donors. Donors 17-18 years had a prevalence of $2.8 \%, 19-24$ years had $2.39 \%$ while donors $25-65$ years had $2.23 \%$ occurrence. Only $2.2 \%$ of their blood donors with adverse effects were at repeat donation. ${ }^{8}$ A report from Javad led study found several adverse effects of blood donation occurring at variable rates; vaso-vagal reactions $2 \%$, arm pain $8.2 \%$, bruise $7 \%$, haematoma $7.4 \%$, and numbness and tingling $0.7 \%$ with no episodes of convulsions among their donors. Regular and repeat donors were found with fewer vaso-vagal symptoms compared to first time donors. ${ }^{9}$ Symvaslakis and others reported increased occurrence of adverse effects of blood donation among adolescents in their study and recommended youth oriented counseling as mitigation strategy. ${ }^{10}$ Studying adverse donor reactions during and immediately after venesection, Mahbub-ul-Alam and colleagues found a 5.04\% prevalence among first time donors and $4.96 \%$ in repeat donors. Their female donors had higher prevalence of $5.97 \%$ while a lower $4.94 \%$ was recorded among the male donors. Vaso vagal effects were at the prevalence of $0.77 \%$ and $0.35 \%$ among female and male donors respectively. ${ }^{11}$ Hosseini and others identified blood donation facilities, personnel, type of blood donation (first time, repeat and frequent donors), age, gender and seasonal variations as predictors of blood donation reactions. ${ }^{12}$ Donic and Lefort enumerated serious adverse effects from collection of blood and blood components to include fainting and seizures and recommended the reporting and management of such occurrences statutory. ${ }^{13}$

There are limited data on the adverse effects of blood donation in our setting. The establishment of the National Blood Service of Nigeria and the attendant increasing pool of voluntary blood donors requires the generation of baseline information for application in the mitigation of adverse donation reactions through donor education, recruitment, counseling and blood collection with the ultimate improvement in quality blood availability and donor satisfaction and retention. This study was to determine the adverse effects of blood donation on voluntary blood givers in Jos North Central Nigeria.

\section{Materials and Method}

This prospective study was carried out at the North Central Zonal Centre of the National Blood Transfusion Service, Jos, Nigeria where ethical approval was obtained. All blood donors recruited by the centre between October 2012 and
September 2014 were counseled and consent to participate in the research was obtained prior to donation. The age, sex, weight and blood pressure were obtained or measured. The haemoglobin level was determined by automation using Haemocue 301 and haemoglobin phenotypes by cellulose acetate electrophoresis. The type of blood donation and the venue of donation were determined and documented. Donors who reacted were observed for the type of donation reaction during and or after donation and timed. Epi info statistical application software version 2007 package was applied for data analysis. The significant value was $\mathrm{P}<0.05$. Results were presented in table and charts.

\section{Results}

A total of 18,500 voluntary blood donors, 11,655 (63\%) males and 6,845 (37\%) females, were studied between October 2012 and September 2014 for adverse effects of blood donation. Three hundred and eighty $(2.05 \%)$ of voluntary blood donors reacted to blood donation during donation or within 30 minutes after, with no case of delayed development of adverse effects. The age distribution of donors were 6747 (36\%), $6456(35 \%), 3651(20 \%)$ and $1646(9 \%)$ for aged $18-$ $25,26-35,36-45$ and $\geq 46$ years respectively. The rate of adverse effects was highest (3.3\%) among donors $18-25$ years compared to $1.8 \%, 0.7 \%$ and $0.9 \%$ respectively for other age groups. This lowering rates with increasing age was significant; $\mathrm{p}<0.0001$ (table 1). Male donors accounted for $160(42 \%)$ reactions while the female counterpart was responsible for 220 $(58 \%)$. The rate of adverse reactions among female donors was $3.2 \%$, significantly higher than $1.4 \%$ among their male counterpart $(\mathrm{p}=0.00001)$. The mean weight of all donors studied was $66.15 \pm 10.38 \mathrm{~kg}$ with a higher $69.27 \pm 10.62 \mathrm{~kg}$ among donors with no donation reactions than a lower $63.03 \pm 9.19 \mathrm{~kg}$ among those who developed adverse effects. The rate of adverse effects of blood donation among voluntary donors was significantly higher (7.6\%) among 106 donors weighing between $40-49 \mathrm{~kg}$ than the higher weight groups; $\mathrm{p}$ $=0.0001$ (table 2).

Table 1. Donor reaction rate among age ranges.

\begin{tabular}{lllll}
\hline Age range & Total No $(\%)$ & $\begin{array}{l}\text { Non reactors } \\
(\%)\end{array}$ & $\begin{array}{l}\text { Reactors } \\
(\%)\end{array}$ & P value \\
\hline 18- 25 years & $6747(36)$ & $6524(96.7)$ & $223(3.3)$ & \\
26- 35 years & $6456(35)$ & $6340(98.2)$ & $116(1.8)$ & 0.00001 \\
36- 45 years & $3651(20)$ & $3625(99.3)$ & $26(0.7)$ & \\
$\geq$ 46 years & $1646(9)$ & $1631(99.1)$ & $15(0.9)$ & \\
Total & $18500(100)$ & $18120(97.95)$ & $380(2.05)$ & \\
\hline
\end{tabular}

Table 2. Donor reaction among weight ranges.

\begin{tabular}{lllll}
\hline $\begin{array}{l}\text { Weight } \\
\text { range }\end{array}$ & Total & Non Reactors (\%) & Reactors (\%) & P value \\
\hline $40-49 \mathrm{Kg}$ & 106 & $98(92.4)$ & $8(7.6)$ & \\
$50-59 \mathrm{Kg}$ & 4004 & $3879(96.9)$ & $125(3.1)$ & 0.0001 \\
$60-69 \mathrm{Kg}$ & 5528 & $5369(97.1)$ & $159(2.9)$ & \\
$70-79 \mathrm{Kg}$ & 4168 & $4099(98.3)$ & $69(1.7)$ & \\
$\geq 80 \mathrm{Kg}$ & 3324 & $3305(99.4)$ & $19(0.6)$ & \\
\hline
\end{tabular}

The systolic and diastolic blood pressures of donors who 
had donation reactions were similar to those who had no adverse effects $(119 \pm 7 / 79 \pm 6 \mathrm{mmHg}$ and $120 \pm 10 / 79 \pm 6$ $\mathrm{mmHg}$ respectively). The mean haemoglobin level of donors with adverse effects was $145.08 \pm 16.92 \mathrm{~g} / \mathrm{L}$. This was similar to $145.51 \pm 16.52 \mathrm{~g} / \mathrm{L}$ mean haemoglobin level of non reactors. Fifty one, 24, 19 and 6 percents of studied blood donors were group $\mathrm{O}, \mathrm{B}, \mathrm{A}$ and $\mathrm{AB}$ respectively. Blood group $\mathrm{B}$ donors in this work constituted the $\mathrm{ABO}$ blood group with the highest (45\%) proportion of reactions while $\mathrm{O}, \mathrm{A}$ and $\mathrm{AB}$ contributed $33 \%, 19 \%$ and $6 \%$ accordingly (figure 1). About $4 \%$ blood group B donors had adverse effects, significantly higher than $1.3 \%, 1.7 \%$ and $2.1 \%$ respectively among groups $\mathrm{O}, \mathrm{A}$ and $\mathrm{AB}$ donors; $\mathrm{p}<0.001$. Three thousand four hundred and sixty one (19.1\%) donors were haemoglobin phenotype AS while the remaining $80.9 \%$ were AA. Adverse effects occurred in $44(1.3 \%)$ of AS donors, significantly lower than $336(2.3 \%)$ adverse event among donors of AA phenotype; $p$ $=0.0002$

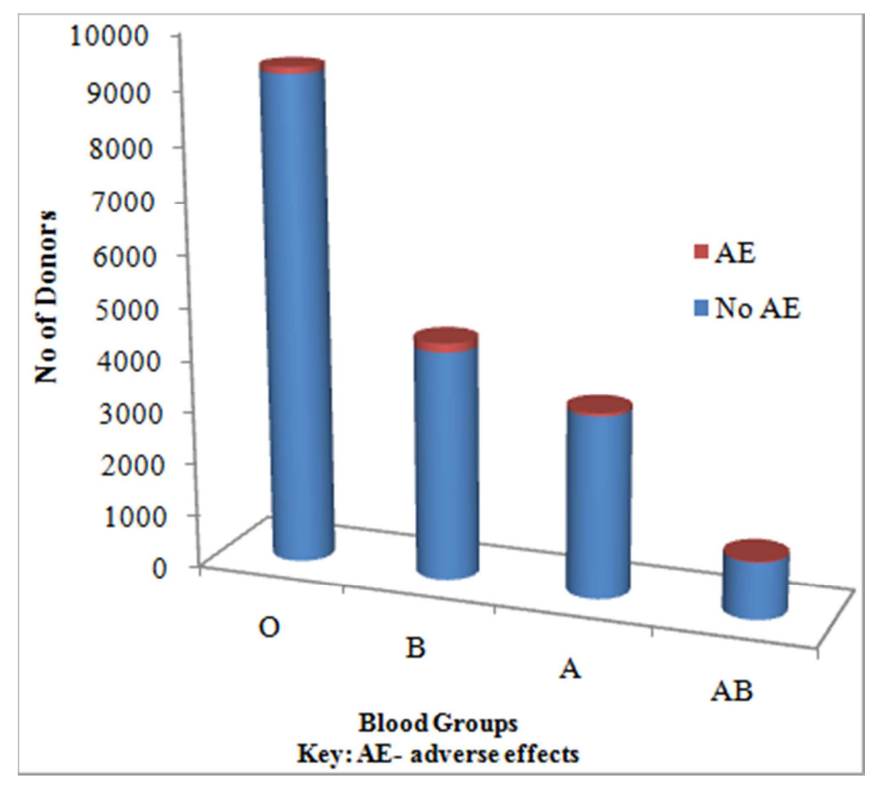

Figure 1. ABO blood group distribution of blood donors.

Five thousand nine hundred and fifty $(32.2 \%)$ of our blood donors were bled indoor while 12550 (67.8\%) were recruited and bled outdoor. The rate of adverse reactions at indoor donations was $2.5 \%$ significantly higher than $1.8 \%$ among the outdoor blood givers; $\mathrm{p}=0.001$. Thirteen thousand three hundred and twenty $(72 \%)$ were first-time donors with an adverse effects of $2.3 \%$ while 5180 (28\%) repeat donors had a significantly lower rate $(1.5 \%)$ of adverse reactions; $p$ $=0.002$.

All adverse effects in our donors occurred between 0-21 minutes of donation $(11.1 \pm 5.62$ mins $)$. Fifteen $(4 \%)$ of them reacted while still donating blood and two hundred and two (53\%) reacted within $1-5$ minutes post donation. One hundred and twenty nine donors reacted within 6-10 minutes after donation while $30(8 \%)$ and $4(1 \%)$ reacted to blood donation in 10-20 and $>20$ minutes respectively (figure 2). The dominant adverse reaction in our donors is dizziness affecting $350(1.9 \%)$ of all donors and $92 \%$ of adverse reactors. Dizziness alone occurred in $81 \%$, while dizziness with vomiting, dizziness with vomiting and faint, dizziness with bleeding and dizziness with faint affected $6 \%, 2 \%, 2 \%$ and $1 \%$ of reactors respectively (figure 3 ). The severe adverse effect (faint) occurred in $0.06 \%$ of all donors, accounting for $3 \%$ of all reactions. All the serious adverse reactions occurred after completion of phlebotomy. Bleeding from the venepuncture site accounted for $30(0.7 \%)$ of all donors and $8 \%$ of reactors in our study. The vomits of $30(8 \%)$ reacting donors who vomited contained recently ingested meals.

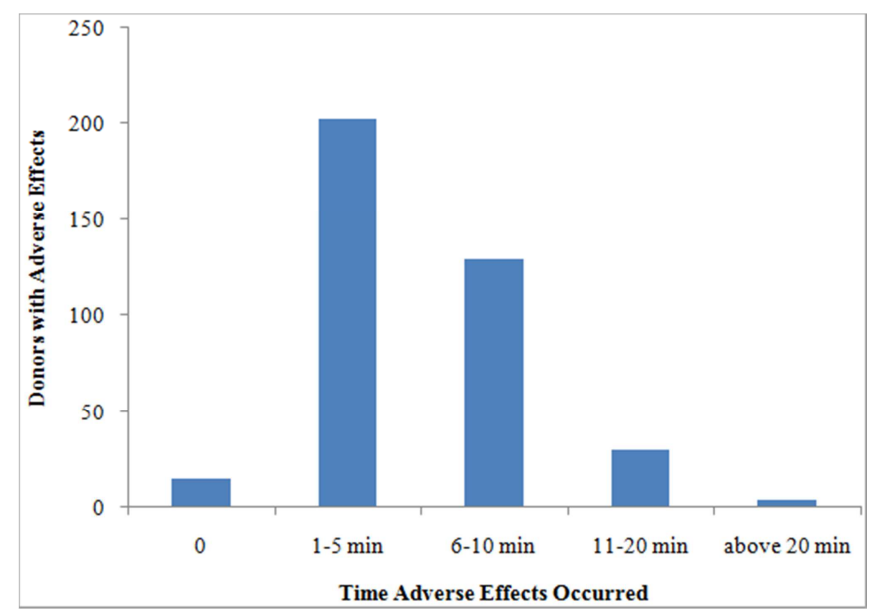

Figure 2. Distribution of timed occurrence of adverse reactions.

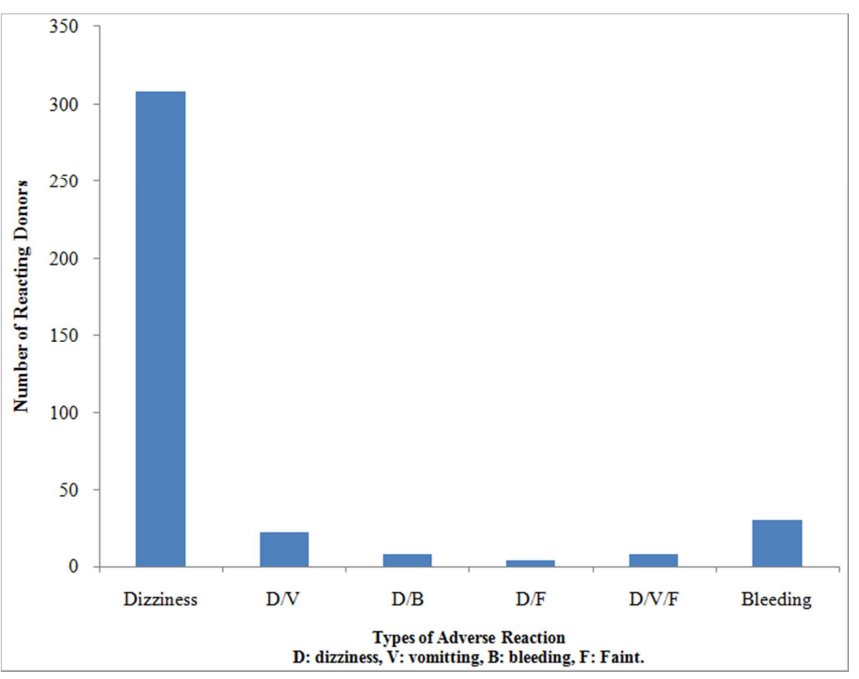

Figure 3. Distribution of adverse reactions.

\section{Discussion}

The rate of occurrence of adverse effects of whole blood donation during and within thirty minutes post donation in our study was $2.05 \%$. This rate is lower than the adverse effects reported at various times by Newman and others (2003, 2004). ${ }^{1,2}$ The overall rate of adverse donation reactions in our work is similar to $1.2 \%$ documented by Crocco et al (2007) among volunteer blood givers in Italy but higher than $0.6 \%$ reported by Pathak et al, among Indian blood donors. ${ }^{5,6}$ The difference in the rate of adverse 
reactions to blood donation between our donors and previous rates documented by other researchers in other climes might be due to the non established reporting system for blood donation reaction in our setting. This is underscored by the absence of off-site reactions in our new centre practicing blood transfusion service on voluntary blood donation. The design of an open blood donation reaction reporting system that involves the donors would capture adverse effects that may occur after the donor had left the blood collection clinic. The age 18-25 year old donors had the highest rate of untoward reactions to blood donation in our centre. The rate of unpleasant experience is similar to $2.8 \%$ reported by Eder et al and Wiltbuk et al among their donors aged above 20 years and $17-18$ years respectively. ${ }^{7,8}$ The $2.05 \%$ rate of adverse effects to blood donation in our study is however lower than $10.7 \%$ and $8.3 \%$ among respectively, $16-17$ and 18-19 years old blood givers included in the Eder's report. ${ }^{7}$ The minimum age requirement of 18 years for blood donation in Nigeria might have excluded a group of younger prospective volunteers at risk of development of adverse effects. Early sensitization and education of teenagers below 18 years old could prepare them for blood donation. The significant higher rate of adverse effects to blood donation in young donors calls careful donor selection, pre and post donation counseling and follow-up in order not to lose young donors to unpleasant donation experiences. Female blood donors in our centre had significant higher rate of adverse effects than their male counterparts similar to higher adverse reactions among female donors reported among subjects in Mahbub-Ul Alam's study. ${ }^{11}$ This calls for keen observation, care and attention to female blood donors in particular, during and after donation to identify early signs of discomfort for immediate prevention and mitigation of evolving adverse effects.

The higher rate of adverse events among whole blood donors aged 18-25 years and those weighing 40-49 kg calls for caution in accepting and collecting blood from the young and underweight donors. The national guideline recommends the minimum age and weight of 18 year and $50 \mathrm{~kg}$ respectively, suitable for whole blood donation. ${ }^{14}$ There is need to introduce component blood donation and whole blood collection in paediatric blood bags to accommodate this category of blood givers. This will increase the donor pool and blood availability while reducing adverse events among young and low weight donors with great potential for long term retention. The similar blood pressures and haemoglobin levels of donors with and those without adverse effects suggest compliance with the clinical state and blood level require for donor selection. The blood pressure of our study group, however suggest overly exclusion of prospective hypertensive blood givers. There is need to follow the national international guideline to capture hypertensive donor with systolic and diastolic blood pressures of $180 / 100 \mathrm{mmHg}$ and below. ${ }^{14}$ This would avail such blood givers the opportunity of correcting possible associated dyslipidaemia, a cardiovascular risk factor associated with hypertension, through regular blood

\section{donation. ${ }^{15,16}$}

Blood donors, who are ABO blood group B and those who are AS haemoglobin phenotype in this study, suffered more adverse effects than other ABO blood groups and AA phenotype. While the association of blood group antigens with diseases and the resistance of haemoglobin AS to plasmodium falciparium infestation have been detamined, ${ }^{17,18}$ we further established a link between occurrence of adverse reactions to blood donation and blood group $\mathrm{B}$ as well as AA haemoglobin phenotype. There is need for further study to confirm and understand these findings. The blood service should use the blood group of donors to selectively give higher attention to donors with $\mathrm{B}$ antigen and or AA haemoglobin phenotype all through the circle of blood donation.

The rate of adverse effects was significantly higher among one third of donor whose donations were in-door. The difference in the rate of adverse effects could be due to the open, interactive and festive atmosphere of outdoor blood donation drives. This suggests the encouragement of out-door blood donation in the community where donating persons could derive support from their peers and the complement of the blood service personnel in a non formal setting. Adverse effects of blood donation occurred more frequently among first-time than repeat donors similar to report by Javad et al who observed higher incidence among first-time donors than regular and repeat donors in their study. ${ }^{9}$ This finding further calls for professionalism in donor recruitment, counseling, phlebotomy and donor care to achieve pleasant donor experiences, hence retention and long term commitment.

The period of occurrence of adverse reaction to blood donation is variable, ranging from pre completion of phlebotomy to less than 30 minutes after. This study indicates that most reactions to blood donation would manifest in our donors in the first ten minutes after donation unlike $24 \%$ delayed reaction reported by Hamal and co-workers. ${ }^{19}$ The incorporation of this information into the protocol for monitoring of adverse effects in blood donors would lead to early detection and mitigation. We suggest the application of the rule of three ten, where the donor is allow to rest in the position of phlebotomy for ten minutes after phlebotomy is completed to accommodate adequate early fluid redistribution, another ten minutes for post donation refreshment while the last ten minutes is utilized for immediate post donation counseling aimed at donor education, encouragement and detection of early features of adverse events. This if done, would provide opportunity for on-site management of unpleasant events while preventing off-site reactions with potential of serious consequences that might be reasons for temporary or in the extreme, permanent self deferral. Continuous commitment to regular donation can also be discussed and offered to the donor during this counseling session.

The adverse reactions observed in our study are similar to that reported by Pathak et al among Indian donors where presyncopal symptoms accounted for $70 \%$ of all adverse reactions. ${ }^{6}$ While Donic and Leffort enumerated faints and 
convulsion among serious effects of blood donation warranting statutory reporting and management, we did not document seizures among our donors. ${ }^{13}$ This may be due to early intervention administered to reacting donors at pre-faint and faint stages on-site, further emphasizing the need to keep donors at venue of donation 30 minutes before departing. The bleeding complication seen in our donors calls for donor education on post donation handling of phlebotomy site. The phlebotomist's keen observation of venepuncture site, after application of adhesive pad, in the early post donation period will prevent or reduce the occurrence of this complication. Recently ingested feeds vomited by some of our donors imply the risk of aspiration of vomits with attendant serious complication that might require intensive care. We strongly recommend that blood donors should not be fed in the period between recruitment and completing phlebotomy to prevent blood shunting to the mesenteric circulation and subsequent vaso-vagal effects. Thus, only post donation refreshment should be given at least ten minutes after completing phlebotomy to reduce vomiting which may discourage commitment to blood donation in the bit to avoid adverse effects due to physiologic changes observed by Gridon. ${ }^{20}$

\section{Conclusion}

There is a predictable low rate of adverse reaction to blood donation among voluntary blood donors. The creation of an informal interactive indoor and outdoor environment for blood donation would probably lead to low rate of adverse events during or soon after blood donation.

\section{Recommendation}

We recommend the development of adverse effect surveillance system and donor educations by all centre that mass recruit and bleed blood donors. We further recommend the inclusion of medical officers in all blood collection exercises, prepared to prevent and manage adverse effects that may occur.

\section{Acknowledgement}

We wish to thank the National Blood Transfusion Service, the Centre for Disease Control and all blood donors for making this work a success.

\section{References}

[1] Newman BH, Pichette S, Pichette D et al. Adverse effects of blood donation. Transfus. 2003; 43: 598-603.

[2] Newman BH. Blood donor complications after whole blood donation. Current Opin Haematol. 2004; 11: 339-345.
[3] Newman BH. Whole blood donation: blood donor suitability and adverse events. Current haematol Rep. 2004; 3:437-443

[4] Newman BH, Roth AJ. Estimating the probability of a blood donation adverse event based on 1000 interviewed whole blood donors. Transfus. 2005; 45: 1715-1721

[5] Crocco A, D'Elia D. Adverse reactions during voluntary donation blood and/or blood components. A statistical epidemiological study. Blood transfus. 2007; 5: 153-152

[6] Pathak C, Pujani M, Pahuja S et al. Adverse reactions in whole blood donors: an Indian scenario. Blood transfuse. 2011; 9: $46-49$

[7] Eder AF, Hillyer CD, Dy BA et al. Adverse reaction to allogeneic whole blood donation by 16 and 17 years old. JAMA. 2008; 299: 2279-2286

[8] Wiltbuk TB, Giordano GF, Kaniel H et al. Faints and prefaints reactions in whole blood donors: an analysis of pre-donation measurements and their predictive value. Transfus. 2008; 48: 1799-1808

[9] Javad H, Shashahani HJZ, Yavari MT. Adverse effects in blood donors after whole blood donation. Sci J Iranian Blood Transfus Org. 2007; 4: 87-93

[10] Symvaslakis EK, Vardavas CL, Fountouli P. Adverse reactions to blood donation among adolescents. JAMA. 2008; 300:1759-1760

[11] Mahbub-ul-Alam M, Hyder MS, Khan MBK et al. Adverse donor reaction during and immediately after venessection. TAJ. 2007; 20: 39-47

[12] Hosseini MJ, Latiff L, Syad Hassan ST et al. Comments on adverse reaction in blood donors. Blood Transfus. 2010; 8: 71

[13] Donic D, Lefort C. Serious adverse effects of blood collection. Transfus Clin Biol. 2010; 17: 301-305

[14] The National Blood Transfusion Service. The National Blood Policy. Federal Ministry of Health Abuja; 2006: 1-24.

[15] Soloren JT, Tuomainen T, Soloren R. Donation of blood is associated with reduced risk of myocardial infarction. AM J Epid. 1998; 48: 445-51

[16] Adediran Uche, E., Adediran, A., Damulak, O.D., Adeyemo, T., Akinbami, A. and Akanmu, A (2013). Lipid profile of regular blood donors. Journal of Blood Medicine. 4; 39-42.

[17] Calhoun L. Petz LD. Erythrocyte antigens and antibodies. In: Ernest B, Marshall AL, Barry SC, Thomas JK, Uri S (edds). Williams Haematology. 6th Edition, McGrwal Hill, New York, 2002: $1843-58$

[18] Beutler E. The sickle cell disease and related disorders. In: Ernest B, Marshall AL, Barry SC, Thomas JK, Uri S (edds). Williams Haematology. 6th Edition, McGrwal Hill, New York, 2002: 581-606.

[19] Kamal H, Tomosolu P, Bravo $M$ et al. delayed adverse reactions to blood donation. Transfus. 2010; 50: 556-65

[20] Gridon AJ. Adverse reactions to whole blood donation and plasmapheresis. Crit Rev Clin Lab Sci. 1982; 17: 51-75 